\title{
Stromal Cell-Derived Factor-1 Antagonizes Slit/Robo Signaling In Vivo
}

\author{
Sreekanth H. Chalasani, ${ }^{1 *}$ Angela Sabol, ${ }^{1 *}$ Hong Xu, ${ }^{1}$ Michael A. Gyda, ${ }^{2}$ Kendall Rasband, ${ }^{3}$ Michael Granato, ${ }^{2}$ \\ Chi-Bin Chien, ${ }^{3}$ and Jonathan A. Raper ${ }^{1}$ \\ ${ }^{1}$ Neuroscience Department and ${ }^{2}$ Cell and Developmental Biology, University of Pennsylvania School of Medicine, Philadelphia, Pennsylvania 19104, and \\ ${ }^{3}$ Department of Neurobiology and Anatomy, University of Utah Medical Center, Salt Lake City, Utah 84132
}

Retinal ganglion cell axons exit the eye, enter the optic stalk, cross the ventral midline at the optic chiasm, and terminate in the optic tectum of the zebrafish. While in the optic stalk, they grow immediately adjacent to cells expressing the powerful retinal axon repellent slit2. The chemokine stromal cell-derived factor-1 (SDF1) is expressed within the optic stalk and its receptor CXCR4 is expressed in retinal ganglion cells. SDF1 makes cultured retinal axons less responsive to slit2. Here, we show that reducing SDF1 signaling in vivo rescues retinal axon pathfinding errors in zebrafish mutants that have a partial functional loss of the slit receptor robo2. In contrast, reducing SDF1 signaling in animals that completely lack the robo2 receptor does not rescue retinal guidance errors. These results demonstrate that endogenous levels of SDF1 antagonize the repellent effects of slit/robo signaling in vivo and that this antagonism is important during axonal pathfinding.

Key words: axon guidance; retinal ganglion cell; zebrafish; SDF-1; slit; astray; modulation

\section{Introduction}

Axonal processes from differentiated neurons are guided to their targets by families of repellent and attractant signaling molecules (Tessier-Lavigne and Goodman, 1996; Yu and Bargmann, 2001). Retinal ganglion cell (RGC) axons are particularly well studied and through a combination of in vitro and genetic studies in a variety of systems have been shown to respond to many guidance cues. These include the attractants laminin and netrin- 1 as well as the repellents slit1, slit2, ephrin As, ephrin Bs, sema 5a, and sema 3d (Cohen et al., 1987; Deiner et al., 1997; Plump et al., 2002; Monschau et al., 1997; Williams et al., 2003; Goldberg et al., 2004; Sakai and Halloran, 2006). Correct pathfinding requires that retinal growth cones appropriately integrate opposing signals. For example, a repulsive cue like slit2 could in principle be antagonized in two distinct ways: first, simple summation with an opposing (attractive) cue; or second, gating by a modulatory signal. The experimental distinction between the two is that in the first case, the attractive cue should still act when the repellent is removed, whereas in the second case, the modulatory cue should have no effect in the absence of repellent signaling. Here, we show that stromal cell-derived factor-1 (SDF1) acts as a modulator to reduce the effects of slit/robo signaling in retinal axons in vivo.

\footnotetext{
Received Sept. 21, 2006; revised Dec. 9, 2006; accepted Dec. 16, 2006.

This work was supported by National Institutes of Health Grant R01-NS26527 to J.A.R. We thank John Kuwada and Qin Li for plasmid constructs, Herwig Baier for the Brn3c:GFP line, and past and current members of the fish facility staff at the University of Pennsylvania.

*S.H.C. and A.S. contributed equally to this work.

Correspondence should be addressed to Jonathan A. Raper, Neuroscience Department, University of Pennsylvania School of Medicine, 1115 BRB II/III, 421 Curie Boulevard, Philadelphia, PA 19104. E-mail: raperj@mail.med.upenn.edu.

DOI:10.1523/JNEUROSCI.4132-06.2007

Copyright $\odot 2007$ Society for Neuroscience $\quad$ 0270-6474/07/270973-08\$15.00/0
}

Slit2 is a powerful repellent of RGC axons in in vitro assays, but surprisingly, retinal ganglion cell axons extend within the optic nerve in close proximity to cells expressing slit2 (Erskine et al., 2000; Niclou et al., 2000). This suggests that slit2 repellent activity is somehow mitigated in the embryonic environment. We recently identified the chemokine SDF1 (CXCL12) as a candidate antirepellent signal. slit2 is a significantly weaker repellent for RGC axons when SDF1 signaling activates the CXCR4 receptor on RGCs (Chalasani et al., 2003a). Conversely, SDF1-induced RGC survival can be blocked by slit signaling (Chalasani et al., 2003b). These findings suggest a mutually antagonistic relationship between SDF1 and slit signaling pathways in vitro. Here, we test whether SDF1 acting via the CXCR4 receptor antagonizes repellent signaling activity during retinal axon pathfinding in vivo.

We have studied the interactions between SDF1 and slit/robo signaling in the developing retinotectal projection of the embryonic zebrafish. Retinal axons first grow toward the optic disc, exit the eye at the ventral fissure, and grow to the ventral midline where they meet and pass axons from the contralateral eye to form the optic chiasm. After crossing the midline, they proceed along the optic tract and project topographically to their target, the optic tectum (Stuermer, 1988; Burrill and Easter, 1994). A screen for genes affecting RGC axon guidance isolated four recessive alleles of the astray (ast) gene in which retinal axons make multiple pathfinding errors. These errors often lead to retinal axons recrossing the midline in ectopic locations after they cross the ventral midline at the optic chiasm (Baier et al., 1996; Karlstrom et al., 1996). The astray gene encodes the slit receptor robo2, the only known robo gene expressed by RGCs during their initial pathfinding (Fricke et al., 2001). Three strong alleles (ast ${ }^{\mathrm{t} i 272 \mathrm{z}}$, ast ${ }^{\mathrm{te} 378}$, and $a s t^{\mathrm{t} 231}$ ) are phenotypically indistinguish- 
able, whereas a fourth, ast $t^{\text {te284 }}$, is detectably weaker. Whereas the ast retinal phenotype varies greatly even within a single clutch of embryos, ast ${ }^{\text {te284 }}$ homozygotes generally display fewer misguided axons and fewer anterior misprojections when compared with the three strong alleles. The ast ${ }^{\text {ti272z }}$ allele encodes a stop codon that truncates robo2 before its transmembrane domain, precluding any receptor function. Ast $t^{\text {te284 }}$ encodes a Gly to Asp missense mutation in the transmembrane domain. Unlike $a s t^{\mathrm{ti272z}}, a s t^{\mathrm{te} 284}$ mutants have normal levels of robo2 mRNA (Fricke et al., 2001). Together, this genetic and molecular evidence shows that ast ${ }^{\mathrm{t} 272 \mathrm{z}}$ mutants lack functional robo2 receptor, whereas ast ${ }^{\text {te284 }}$ mutants are hypomorphs and have a low level of residual robo2 function.

Genes encoding two SDF1 homologues, $s d f 1 a$ and $s d f 1 b$, and two CXCR4 homologues, cxcr4a and cxcr $4 b$, have been identified in zebrafish (Chong et al., 2001; Doitsidou et al., 2002). Cxcr4b, but not $\operatorname{cxcr} 4 a$, is specifically expressed by RGCs even before they extend axons (Chong et al., 2001). Both $s d f 1 a$ and $s d f 1 b$ are expressed in the optic stalk (Li et al., 2005). Antisense morpholinos are a well characterized method for knocking down gene function in zebrafish (Nasevicius and Ekker, 2000) and have been used to make effective knockdowns of both SDF1 and CXCR4. Morpholinos against SDF1 and/or CXCR4 have shown that this signaling system plays an essential role in the guidance of germ cells to the gonads (Doitsidou et al., 2002; Knaut et al., 2003), sensory cell migration along the lateral line ( $\mathrm{Li}$ et al., 2004), lateral line axon guidance (David et al., 2002), and retinal axon extension within the eye (Li et al., 2005).

Experiments with cultured primary neurons demonstrate a mutual antagonism between SDF1 and slit signaling pathways. Our aim was to test whether the same is true during normal development in vivo. We hypothesized that residual slit/robo2 signaling in ast ${ }^{\text {te284 }}$ embryos should be rendered more effective if SDF1/CXCR4 signaling were reduced. If so, the resulting enhanced effectiveness of slit/robo 2 signaling would be predicted to rescue retinal guidance defects in $a s t^{\text {te284 }}$ larvae. We used morpholinos to knock down either CXCR4 or SDF1 in ast ${ }^{\text {te284 }}$ embryos in which there is a partial loss of robo2 function, or in $a s t^{\mathrm{t} 272 \mathrm{z}}$ embryos in which there is a full loss of robo2 function. We find that reduced SDF1/CXCR4 signaling tends to rescue retinal guidance errors in fish with a partial loss of robo2 function. The same manipulations do not effect a rescue of retinal pathfinding errors in fish with a complete loss of robo2 function. These results are consistent with SDF/CXCR4 signaling mitigating the repellent effects of slit/robo signaling during normal development of the visual pathway in vivo.

\section{Materials and Methods}

Zebrafish maintenance. Zebrafish were raised and maintained as described previously (Mullins et al., 1994). Zebrafish embryos were collected at the one-cell stage and kept at $28.5^{\circ} \mathrm{C}$ in $1 \times \mathrm{E} 3$ solution [containing the following (in mM): $5 \mathrm{NaCL}, 0.17 \mathrm{KCl}, 0.33 \mathrm{CaCl}_{2}, 0.33 \mathrm{MgSO}_{4}$ in deionized water]. At 24 hours postfertilization (hpf), collected embryos were sustained in $0.006 \%$ PTU ( $N$-phenylthiourea; P-7629; Sigma, St. Louis, MO) in $1 \times$ E3 solution to help prevent pigmentation. Zebrafish astray te284 and ti272z adult homozygotes are viable and fertile (Fricke et al., 2001). Transgenic $\operatorname{Tg}(b r n 3 c: g f p)^{s 356 t}$ fish (a generous gift from Herwig Baier, University of California, San Francisco, CA) (Xiao et al., 2005) were mated with te 284 astray fish to produce transgenic mutant lines. For most experiments, homozygous astray fish were mated with one another to produce eggs for morpholino injection experiments. In some experiments, fish heterozygous for both astray ${ }^{\text {te284 }}$ and for $b r n 3 c$ : $g f p$ were mated with one another. Homozygous astray fish were identified by PCR using the primers $5^{\prime}$-TACAGAGAACGTGTGCTTCC and $5^{\prime}$-GGGGCCTGCTGGGTCATTCTGATCG (annealed at $60^{\circ}$ ) that am- plify a $153 \mathrm{bp}$ band that is not cut by TaqI in wild-type (WT) fish, but is cut into 128 and 25 bp fragments in astray fish.

Zebrafish retinal cultures. Eyes were dissected from $48 \mathrm{hpf}$ larvae and cultures from the neural retina were established. Explants were plated onto laminin-coated glass coverslips in medium composed of $67 \% \mathrm{~L} 15$ that had been supplemented with 1:50 B27 (17504-044; Invitrogen, Eugene, OR), $6 \mathrm{mg} / \mathrm{ml}$ glucose, $2 \mathrm{~mm}$ glutamine, $100 \mu \mathrm{M}$ putrescine, $200 \mu \mathrm{M}$ progesterone, $5 \mu \mathrm{g} / \mathrm{ml}$ insulin, $20 \mu \mathrm{g} / \mathrm{ml} \mathrm{NGF}, 500 \mu \mathrm{l}$ bovine pituitary extract per $100 \mathrm{ml}$ medium, $100 \mathrm{U} / \mathrm{ml}$ penicillin, $100 \mu \mathrm{g} / \mathrm{ml}$ streptomycin, and $50 \mu \mathrm{g} / \mathrm{ml}$ gentamycin. Human slit 2 and zebrafish SDF1a were made as secreted proteins from transient transfections of $293 \mathrm{~T}$ cells cultured in L15 medium supplemented with 10\% FBS. Crude supernatants were diluted into culture medium. Retinal axons growing out of these explants were treated with (1) 30\% mock transfected (empty plasmid), (2) $15 \%$ mock transfected plus $15 \%$ human slit2-transfected supernatant, (3) or 15\% slit2 plus 15\% zebrafish SDF1-transfected supernatant. A CCD camera (Spot; Phase 3 Imaging, Glen Mills, PA) was used to capture images that were then analyzed using Image-Pro software.

Morpholino injections. Morpholinos (MOs) were obtained from Gene Tools (Bhilomath, OR) and were injected into embryo yolk immediately after fertilization before cell division began. MO sequences for various genes are as follows: CXCR4b, AAATGATGCTATCGTAAAATTCCAT (6 ng/embryo); SDFla, TTGAGATCCATGTTTGCAGTGTGAA (3 ng/ embryo); SDF1B, CGCTACTACTTTGCTATCCATGCCA (3 ng/embryo); control MO, CCTCTTACCTCAGTTACAATTTATA (6 ng/embryo).

All MOs were designed to be translation blocking and were injected at a volume of $0.5 \mathrm{~nL}$ per embryo.

DiI/DiO labeling. Zebrafish larvae at $120 \mathrm{hpf}$ were fixed in 4\% paraformaldehyde (PFA) and stabilized in $1.5 \%$ agarose for injection. Lipophilic dyes $1,1^{\prime}$-dioctadecyl-3,3,3',3'-tetramethylindocarbocyanine perchlorate (DiI) (D-282; Invitrogen) and 3,3'-dioctadecyloxacarbocyanine perchlorate (DiO) (D-275; Invitrogen) dissolved at $2 \%$ in chloroform were injected. After injection, the dyes were allowed to diffuse toward the tectum overnight at room temperature, after which the embryos were assayed for errors in retinotectal pathfinding on a Zeiss (Oberkochen, Germany) Axiovert35 fluorescent microscope. Images were obtained on a Leica (Nussloch, Germany) DMIRE2 confocal microscope after removing the eyes.

Immunostaining. Larvae at various ages were fixed overnight at $4^{\circ} \mathrm{C}$ in $4 \%$ PFA in $0.1 \mathrm{M}$ phosphate buffer plus $1 \%$ DMSO. Collagenase digestion (0.2\% in $1 \times$ PBS for between 1:00 and 1:45 h) (17018029; Invitrogen) was used to facilitate antibody access into the tissues. A monoclonal anti-GFP antibody JL-8 (1:100; 8371-2; BD Biosciences, Franklin Lakes, NJ) followed by an anti-mouse IgG Alexa Fluor 488 (1:400; Invitrogen) was used to visualize green fluorescent protein (GFP)-positive RGC axons. Stained larvae were then transferred to Vectashield mounting medium for imaging using a Leica DMIRE2 confocal microscope. A colorcoded projection that allows the visualization of depth on the dorsalventral axes was created using software supplied with the Leica confocal microscope. A stack of images from the dorsal-most plane of RGC axon projection to the ventral-most plane was first taken. The projection of all of these images was then made and color-coded according to the plane in which the different objects first appeared. The ventral-most plane was given a purple color whereas the dorsal-most plane was identified by red.

Whole-mount fluorescent in situ hybridization. Zebrafish larvae at the indicated times were fixed in 4\% PFA and then treated with collagenase before processing for in situ hybridization. Expression patterns for various transcripts were detected using digoxigenin-labeled antisense probes. The signal was amplified using a fluorescein-coupled tyramide system (TSA Fluorescence System; NEL701A; Perkin-Elmer, Wellesley, MA). Retinal axons were identified in $48 \mathrm{hpflarvae}$ using a mouse monoclonal anti- $\alpha$ acetylated tubulin (1:800; T6793; Sigma) and in $72 \mathrm{hpf}$ larvae with mouse monoclonal anti-GFP antibody JL-8 (1:100; 8371-2; BD Biosciences). These antibodies were detected using an anti-mouse IgG Alexa Fluor 546 (1:400; Invitrogen). Animals were then transferred to Vectashield mounting medium (H-1000; Vector labs, Burlingame, CA) for imaging using a Leica DMIRE2 confocal microscope. 


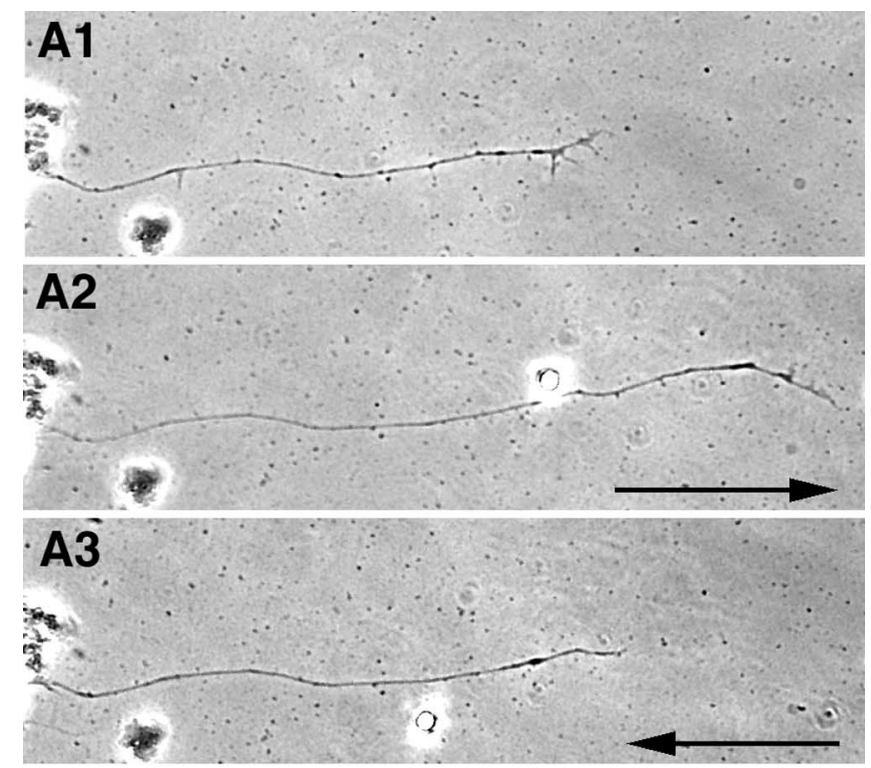

B

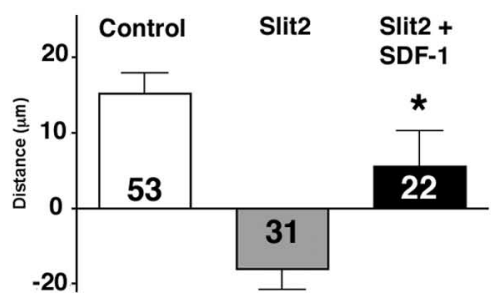

Figure 1. SDF1 reduces slit2 repellent activity on cultured zebrafish retinal axons. $\mathbf{A 1}, \mathbf{A 2}$, Retinal neurons extend axons in culture that advance during a $1.5 \mathrm{~h}$ control period. Arrow, +39 $\mu \mathrm{m}$. A3, Advance is reversed in the presence of hslit2. Arrow, $-33 \mu \mathrm{m}$. $\boldsymbol{B}$, The average distance growth cones extend during the control period (open bar) is compared with extension during treatment with hslit2 (gray bar) or during treatment with both hslit2 and zSDF1a (dark bar). Axons tend to halt or retreat when treated with hslit2 but on average advance in the presence of both hslit2 and ZSDF1a. The SEM and the numbers of growth cones examined in each condition are shown. The average distances of extension during slit2 treatment as compared with slit2 plus SDF1a treatment were significantly different (asterisks) to the $p<0.05$ by the Mann-Whitney test. Error bars indicate SEM.

\section{Results}

Zebrafish SDF1 reduces the effectiveness of slit2 on retinal axons growing in vitro

Previous work has shown that SDF1 antagonizes the repellent effects of slit2 on chick retinal ganglion cell axons (Chalasani et al., 2003a). Retinal explant cultures were established from $48 \mathrm{hpf}$ zebrafish larvae to test whether SDF1a antagonizes the repellent effects of slit2 in zebrafish RGC axons. Retinal axon outgrowth was observed from explants cultured for $20 \mathrm{~h}$. The distances that individual growth cones traveled during a $1.5 \mathrm{~h}$ control period and a subsequent $1.5 \mathrm{~h}$ experimental period were compared. Cultures were exposed to mock-transfected medium during the control period. During the experimental period, they were exposed either to (1) medium collected from slit2 and from mock transfected cells, (2) media collected from slit 2 and from SDF1a expressing cells, or (3) medium collected from SDF1a and mock transfected cells. On average, axons extended forward during the control period (Fig. 1A1,A2). slit2 halted their progress and frequently induced axon retraction, leading to an average net retreat (Fig. A2,A3). The simultaneous application of SDF1a attenuated the activity of slit2 (Fig. $1 B$ ). The addition of SDF1a alone had no
A
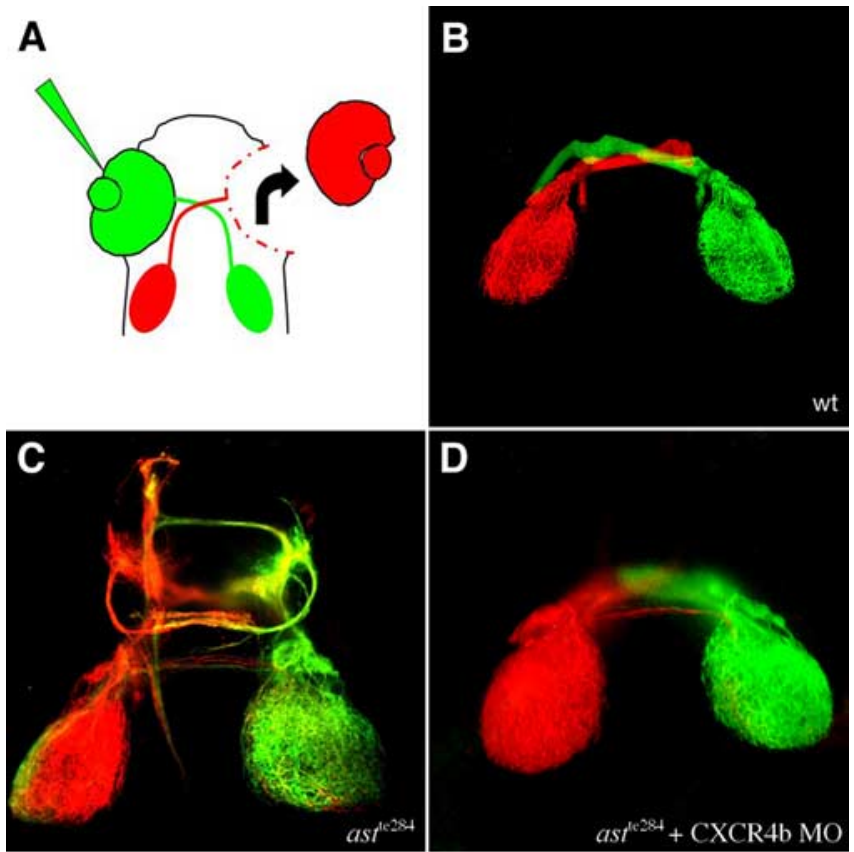

E

$\mathbf{F}$
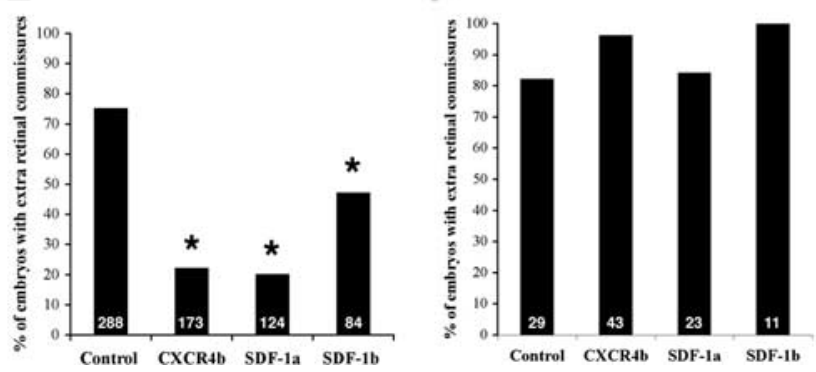

Figure 2. Morpholinos against SDF1 or CXCR4b rescue retinal axon guidance defects in ast ${ }^{\text {te284 }}$ embryos but not ast ${ }^{\text {tiz72z }}$ embryos. $A$, Retinal ganglion cells were labeled with Dil or Di0 injection into each eye. The eyes were removed to facilitate visualization of their axonal projections. $\boldsymbol{B}-\boldsymbol{D}$, Example projection patterns from $120 \mathrm{hpf}$ WT larvae $(\boldsymbol{B})$, ast ${ }^{\text {te } 284}$ larvae $(\boldsymbol{C}$, and ast $^{\text {te284 }}$ larvae (D) after injection of CXCR4b morpholinos. Projections in ast ${ }^{\text {te284 }}$ larvae are characterized by numerous guidance errors including extra crossings, anterior and posterior misprojections and some ipsilateral misprojections. Some but not all of these defects are rescued by CXCR4b morpholinos. Scale bar, $100 \mu \mathrm{m}$. $\boldsymbol{E}$, The percentage of eye projections with extra ectopic commissures is reduced by morpholinos against either SDF1a or CXCR4b injected into ast ${ }^{\text {te284 }}$ embryos that have partial robo2 function. Morpholinos against SDF1b are less effective. The results for all three morpholinos are significantly different from control conditions by Fisher's exact test. ${ }^{*} p<0.001$. $\boldsymbol{F}$, The same morpholinos effect no rescue in astray ${ }^{\text {tiz }}$.2z larvae that have no robo2 function.

significant effect on RGC axon advance (data not shown). These findings demonstrate that zebrafish SDFla antagonizes the repellent effect of slit2 and has no apparent outgrowth accelerating effect of its own on retinal axons in vitro.

\section{Morpholinos against CXCR4 or SDF1 rescue retinal axon defects in astray hypomorphs}

We next tested whether reducing SDF1/CXCR4 signaling can rescue retinal guidance defects in fish with a partial loss of robo2 function. In a first round of experiments, retinal projections were visualized in $120 \mathrm{hpf}$ morphant larvae by labeling axons from one eye with DiI and its contralateral partner with DiO (Fig. 2A). Retinal axons project to the contralateral optic tectum in wildtype larvae within a coherent bundle (Fig. 2 B). Retinal axon trajectories in ast $t^{\text {te284 }}$ larvae have multiple defects that generally arise at the ventral midline or after crossing the midline. These 
include defasciculation, abnormal anterior branches, and ectopic anterior and posterior commissural fascicles (Fricke et al., 2001) (Fig. 2C). To quantify phenotypic rescue, we used a conservative measure, scoring all eyes with ectopic retinal commissures as abnormal. Injection of morpholinos against either CXCR4b or SDF1a into just fertilized ast ${ }^{\text {te284 }}$ eggs (morphants) induces a dramatic rescue of guidance defects as judged by the presence or absence of ectopic commissures in $120 \mathrm{hpf}$ larvae (Fig. 2D,E). The rescue achieved by these morpholinos was statistically significant to a high level. Knock-down of SDF1b is less effective in rescuing defects in retinal trajectories (Fig. $2 E$ ).

\section{Morpholinos against SDF1 or CXCR4 do not rescue retinal guidance defects in astray mutants lacking robo2}

If knocking down SDF1/CXCR4 rescues defects in ast ${ }^{\text {te284 }}$ larvae that have a partial loss of robo2 function through the enhancement of residual slit/robo signaling, then the same manipulation should fail to rescue defects in $a s t^{\mathrm{ti} 272 \mathrm{z}}$ larvae in which there is no functional robo2. Morpholinos against SDF1a or CXCR4b injected into the null mutant ast ${ }^{\mathrm{t} 272 \mathrm{z}}$ produced no discernable rescue of retinal pathfinding errors as assayed by counting ectopic commissures in $120 \mathrm{hpf}$ larvae (Fig. 2 F). Just as reported previously in wild-type embryos, morpholinos against SDF1a or CXCR4b injected into ast ${ }^{\mathrm{t} 272 \mathrm{z}}$ mutants occasionally interfered with the ability of retinal axons to exit the eye through the optic stalk (Li et al., 2005) and frequently induced abnormal and shortened axonal trajectories in the lateral line nerve (David et al., 2002; Li et al., 2004). These observations demonstrate that the morpholinos were effective, but even so, they only rescued retinal pathfinding errors when there was residual robo2 function.

\section{Expression patterns of slits and SDFs suggest regions where the two signals could interact in the retinotectal pathway} The expression patterns of the four zebrafish slit and two $s d f$ genes were compared at different times during embryonic development to identify those whose signaling pathways are most likely to interact. Retinal axons have crossed the midline at $48 \mathrm{hpf}$, entered the optic tract, and grown dorsally toward the optic tectum (Stuermer, 1988; Burrill and Easter, 1994). RGCs express messenger RNAs for both the slit receptor robo2 (Fig. $3 A$ ) and the SDF1 receptor cxcr $4 b$ (Fig. $3 B$ ), consistent with the finding in culture that retinal axons respond to slit2 and to SDF1a. slit2 (Fig. $3 C$ ) and SDF1a (Fig. 3D) are expressed within the optic stalk and surrounding the optic nerve where RGC axons enter the brain (Fig. 3C,D, insets). This suggests that the stalk is a likely locus of interaction between the two signals. Slit1a, slit1b, and slit 3 were not detected in the optic stalk, however they are all strongly expressed along with slit 2 at the ventral midline posterior to the retinal projection (Fig. $3 E-H$ ). Strong slit $1 a$ and slit2 expression are also observed in bilaterally symmetric patches well anterior to the chiasm (Fig. $3 E, G$ ). SDF1a is expressed until $\sim 48 \mathrm{hpf}$ at the edge of the CNS nearby but ventral to these clusters of slit expression (Fig. 3I), suggesting a second locus where the two signals could interact. The other SDF1 homolog, SDF1b, is most strongly expressed posteriorly and well dorsal to the optic chiasm (Fig. $3 J)$. The difference in strength of rescue affected by SDF1a and SDF1b morpholinos (Fig. 2E) is likely explained by the much lower expression of SDF1b in the optic pathway.

\section{Astray axons project inappropriately into regions of slit expression}

A transgenic line in which the $b r n 3 c$ promoter drives GFP in RGCs was used to examine the relationship between RGC axon trajectories and slit expression patterns (Xiao et al., 2005). RGC axons in $54 \mathrm{hpf}$ WT larvae normally do not approach slit2expressing areas anterior to the chiasm (Fig. $4 A, C 1$ ). In contrast, axons in ast $^{\text {te } 284}$ fish sometimes wander anteriorward after they cross the ventral midline and come into close proximity to the slit2 expressing areas (Fig. 4B,D1). A confocal projection that is color-coded by dorsal-ventral position shows that RGC axons normally extend at a distance from slit2 expressing areas (Fig. $4 C 2$ ), whereas in contrast, astray axons grow closer and just ventral to them (Fig. 4D2). Similarly, RGC axons do not normally grow near slit1a-expressing patches anterior to the chiasm (Fig. $4 E 1, E 2)$, whereas ast $t^{\text {te284 }}$ axons sometimes extend just ventral to them (Fig. 4F1,F2). slit2 and slit1a expression anterior to the chiasm might therefore help prevent retinal axons from misprojecting along abnormal anterior pathways. Reduced slit/robo signaling in astray retinal axons is likely to interfere with this anterior repellent signal and account for abnormal anterior projections.

\section{Reduced SDF1 signaling rescues a specific class of retinal axon defects in mutants with a partial loss of robo2}

We next analyzed ast $t^{\text {te284 }}$ larvae injected with morpholinos against SDF1a in more detail to determine whether ectopic anterior projections are specifically rescued when SDF1 signaling is blocked. After crossing the ventral midline, retinal axons from 72 hpf astray larvae make numerous guidance errors both anterior and posterior to the optic chiasm (Fig. $5 A$ ). Interfering with SDF1 signaling reduces defects anterior to the chiasm. (Fig. $5 B, E$ ). However, ectopic dorsal commissures between the two tecta were not rescued (Fig. 5E). Similar experiments were also performed with $a s t^{\text {ti272z }}$ larvae that lack robo2. Defects in RGC projections were observed both anterior and posterior to the optic chiasm (Fig. 5C). Morpholinos against SDF1a did not reduce the frequency of any of these abnormal projections (Fig. $5 D, F$ ). We were surprised to find that a significant proportion of SDF-1a morphants still had an extra dorsal tectal commissure at $72 \mathrm{hpf}$ as compared with their apparent absence in our first round of experiments in which we analyzed morphants at $120 \mathrm{hpf}$. One possible explanation is that the dorsal commissure is too thin to be reliably detected in the older and larger brains using traditional fluorescent optics, but can be visualized with the confocal optics that we used on the younger larvae. Alternatively, the dorsal tectal commissures might be withdrawn in morphants during the period between 72 and $120 \mathrm{hpf}$. These results demonstrate that anterior misprojections of retinal axons in larvae with partial loss of robo2 function are specifically rescued in SDF1a morphants. The lack of rescue in robo2-null mutants suggests that at least some residual slit signaling is required for SDF1 to exert its effects.

Our studies highlight the importance of slits, SDF1, and interactions between them in retinal axon pathfinding in vivo. In wildtype fish, RGCs express the slit receptor robo2 and the SDF1 receptor CXCR4b. Their axons (Fig. $6 \mathrm{~A}$, navy blue) exit the eye and project along the optic pathway to their target, the tectum. They extend in close proximity to slits (Fig. 6, shades of red) and SDF1 (Fig. 6, green). After crossing the midline, retinal axons grow in a posterior and dorsal path toward their target, avoiding anterior concentrations of slit expression. In mutant fish with reduced slit signaling (Fig. 6B, blue), some retinal axons travel forward very near these regions of high slit expression. Their inability to respond normally to slits likely explains their anterior guidance defects. Reduced SDF1 signaling tends to correct anterior retinal pathfinding errors in fish with reduced slit/robo sig- 

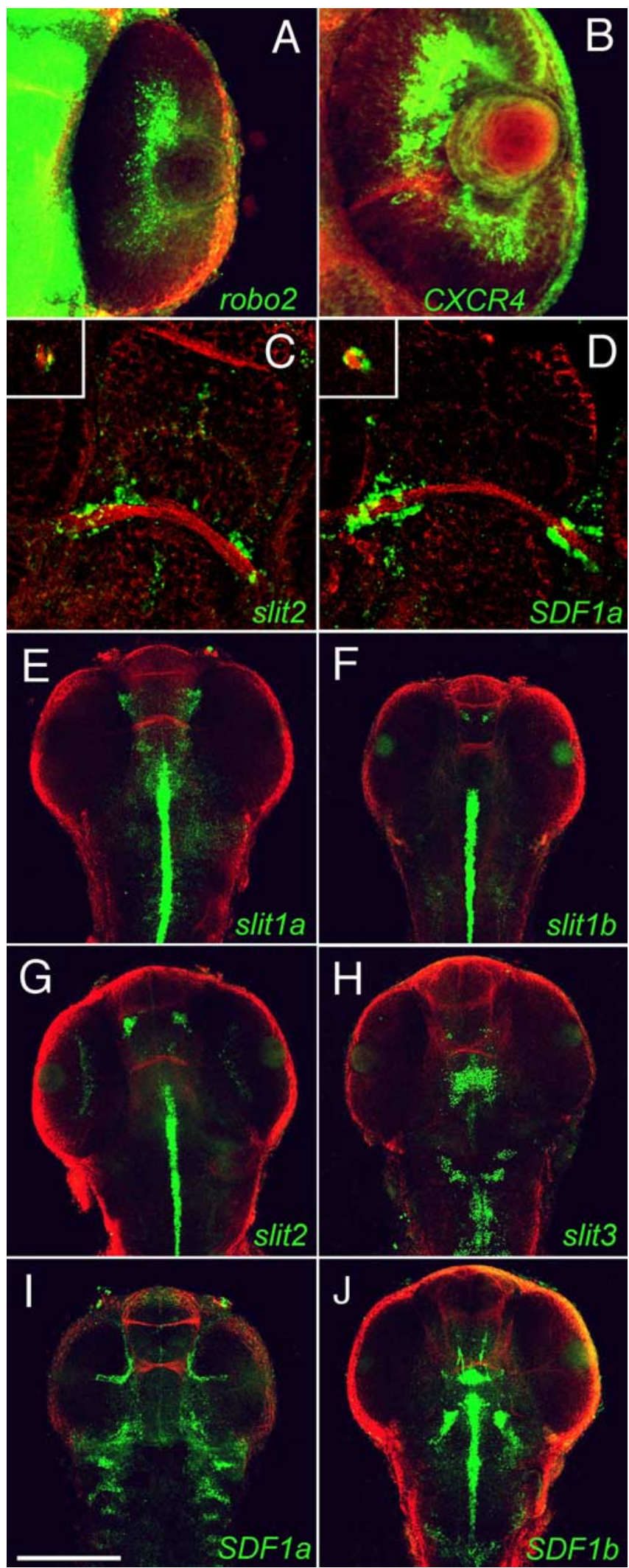

Figure 3. The spatial expression patterns of slits, SDFs, and their receptors along the retinotectal pathway at $48 \mathrm{hpf}$. Projections made from confocal images of the in situ expression of mRNAs (green) and of axons labeled with antibodies against acetylated $\alpha$-tubulin (red) are shown. The prominent rostral pathway across the midline is the anterior commissure. $\boldsymbol{A}, \boldsymbol{B}$ Retinal ganglion cells in the eye express both the slit receptor robo2 $(\boldsymbol{A})$ and the SDF-1 receptor CXCR4b (B). C, D, Projections of confocal images collected from the ventral sides of embryos show that slit2 (C) and SDF1a (D) are expressed at the edge of the CNS where retinal axons naling (Fig. 6C), but not in fish in which slit/robo signaling is entirely absent (Fig. 6D).

\section{Discussion}

slit2 has been shown to act as a powerful repellent on chick, mouse, and now zebrafish retinal axons in vitro (Erskine et al. 2000; Niclou et al., 2000) (Fig. 1). Surprisingly, slit2 mRNA is expressed in the optic stalks of mice very near growing retinal axons (Erskine et al., 2000; Niclou et al., 2000), and similarly, slit2 is expressed in the stalks of developing zebrafish at the same time that axons are growing through them (Hutson and Chien, 2002) (Fig. 3C). How is it possible that axons can extend through what appears to be a hostile environment? One attractive explanation is that countervailing signals within the stalk antagonize slit repellent activity. In tissue culture, the presence of SDF1 makes retinal axons less responsive to slit 2 and other repellents through a signaling pathway that induces elevated levels of cAMP, activation of protein kinase $\mathrm{A}$, and at least partially through the inactivation of Rho (Chalasani et al., 2003a). Similar to our previous findings in vitro using chick and mouse cultures, slit 2 induces the paralysis or retraction of cultured zebrafish RGC axons. This response is greatly reduced in the presence of SDF1a. If SDF1a were an attractant analogous to netrin or one of the neurotrophins, it might be expected to augment retinal axon outgrowth in vitro (Cohen et al., 1994; Deiner et al., 1997), but by itself, SDF1a did not have a detectable outgrowth-inducing effect in our in vitro experiments. Our in vitro findings are consistent with SDF1a making zebrafish retinal axons resistant to the repellent effects of slit2.

Just after retinal axons cross the midline at the optic chiasm, they grow laterally and then dorsally toward the tecta. In astray embryos that have reduced robo 2 function, they are more likely to extend anteriorly along ectopic routes. The first signs of abnormal anterior trajectories are sometimes evident as early as the chiasm, are more prominent just past the chiasm as axons grow across the ventral surface of the diencephalon, and most prominent as they extend sharply anterior ward along the ventrolateral edge of the CNS. These errors in the absence of robo 2 suggest that slit $1 \mathrm{a}$, slit $1 \mathrm{~b}$, and slit 2 expression in bilateral patches just anterior to the optic pathway are important repellent centers for retinal axons that help to direct them into their normal more posterior and dorsal trajectory.

Simultaneously reducing SDF1 signaling rescues retinal axon pathfinding errors in fish that have a partial loss of robo2 function. Rescue is specific to the SDF1 signaling pathway as demonstrated by the finding that morpholinos against either SDF1a or its receptor CXCR4b are equally potent in effecting rescue. In particular, the abnormal sharp anteriorward extension of retinal axons after they have crossed the ventral midline are less frequent in embryos with partial loss of robo 2 function and reduced SDF1a signaling as compared with partial loss of robo2 alone. In

$\leftarrow$

approach the optic chiasm and around the edge of the optic stalk (insets). $\boldsymbol{E}-\boldsymbol{H}$, Projections of confocal images collected from the dorsal sides of embryos show that slit 1a is expressed in the midline posterior to the chiasm and in lateral CNS regions anterior to the chiasm $(\boldsymbol{E})$, slit $1 b$ is expressed at the midline posterior to the optic projection and in two small bilaterally symmetric clusters of cells anterior to the optic chiasm $(\boldsymbol{F})$; slit2 is expressed at the midline posterior to the optic projection and in two prominent bilaterally symmetric clusters of cells anterior to the chiasm (G); and slit3 is expressed predominantly posterior to the chiasm $(\boldsymbol{H}) . \boldsymbol{I}$, SDF1a is expressed in the optic stalk and at the lateral margin of the CNS. J, SDF1 $b$ is strongly expressed at the midline posterior and well dorsal to the optic pathway. Scale bar: (in I) $\boldsymbol{A}, \boldsymbol{B}, 100 \mu \mathrm{m} ; \boldsymbol{C}, \boldsymbol{D}$, $50 \mu \mathrm{m} ; \boldsymbol{E}-\boldsymbol{J}, 250 \mu \mathrm{m}$. 
the region in which these anterior errors occur, SDF1a is not expressed in the same locations as the slits, but instead along the surface of the CNS nearby. There are potentially two explanations for how the rescue is accomplished. In one, SDF1a acts as a traditional attractant that draws retinal axons forward along the edge of the CNS, whereas in the other, it acts as an antirepellent that allows retinal axons to penetrate anteriorly into regions with reduced repellent activity.

There is a strong precedent for SDF1a acting as a traditional attractant for motile cells. SDF1 was initially identified as an attractant for $T$ cells in the immune system (Nagasawa et al., 1994). In zebrafish, SDF1 signaling has been shown to attract primordial germ cells to the gonads and lateral line primordia to their destinations (Doitsidou et al., 2002; Knaut et al., 2003; Li et al., 2004). Zebrafish RGC axons have been reported to alter their trajectories to approach ectopic sites of SDF1a expression (Li et al., 2005). Retinal axons extend within the eye in aberrant fascicles in either ody embryos (in which CXCR4b is absent) or in embryos treated simultaneously with morpholinos against SDF1a and CXCR4b (Li et al., 2005) (Fig. 6C). These studies demonstrate that SDF1/ CXCR4 signaling plays an essential role in normal retinal pathfinding. Misguidance of retinal axons within the eye in the absence of SDF1 signaling could, in principle, again be explained by SDF1a acting as either an attractant or an antirepellent. SDF1 expressed in the optic stalk could either help draw retinal axons into the stalk through an attractive mechanism, or prevent repellents like slit2 in the stalk from excluding retinal axons through an antirepellent mechanism. In preliminary experiments, we observed ectopic retinal axon exits induced at a low frequency by the combination of SDF1a and CXCR4b morpholinos in both phenotypically $a s t^{\mathrm{ti} 272 \mathrm{z}}-1+$ and phenotypically $a s t^{\mathrm{t} 272 \mathrm{z}}$ $-/-$ embryos. This result does not support the notion that SDF1a allows retinal axons to enter the stalk by making them less responsive to slits. These results indicate that SDF1 is acting in the eye either as a traditional attractant for retinal axons into the stalk, or that it induces ectopic exits by mitigating the effectiveness of some other repellent factor, perhaps Semaphorin 5a (Goldberg et al., 2004), that is distinct from slit2.

The rescue of anterior misprojections within the brain obtained by knocking down SDF1a in embryos with a partial loss of robo 2 could be explained if SDF1a has retinal attractant activity that misguides retinal axons in the absence of normal slit signal-

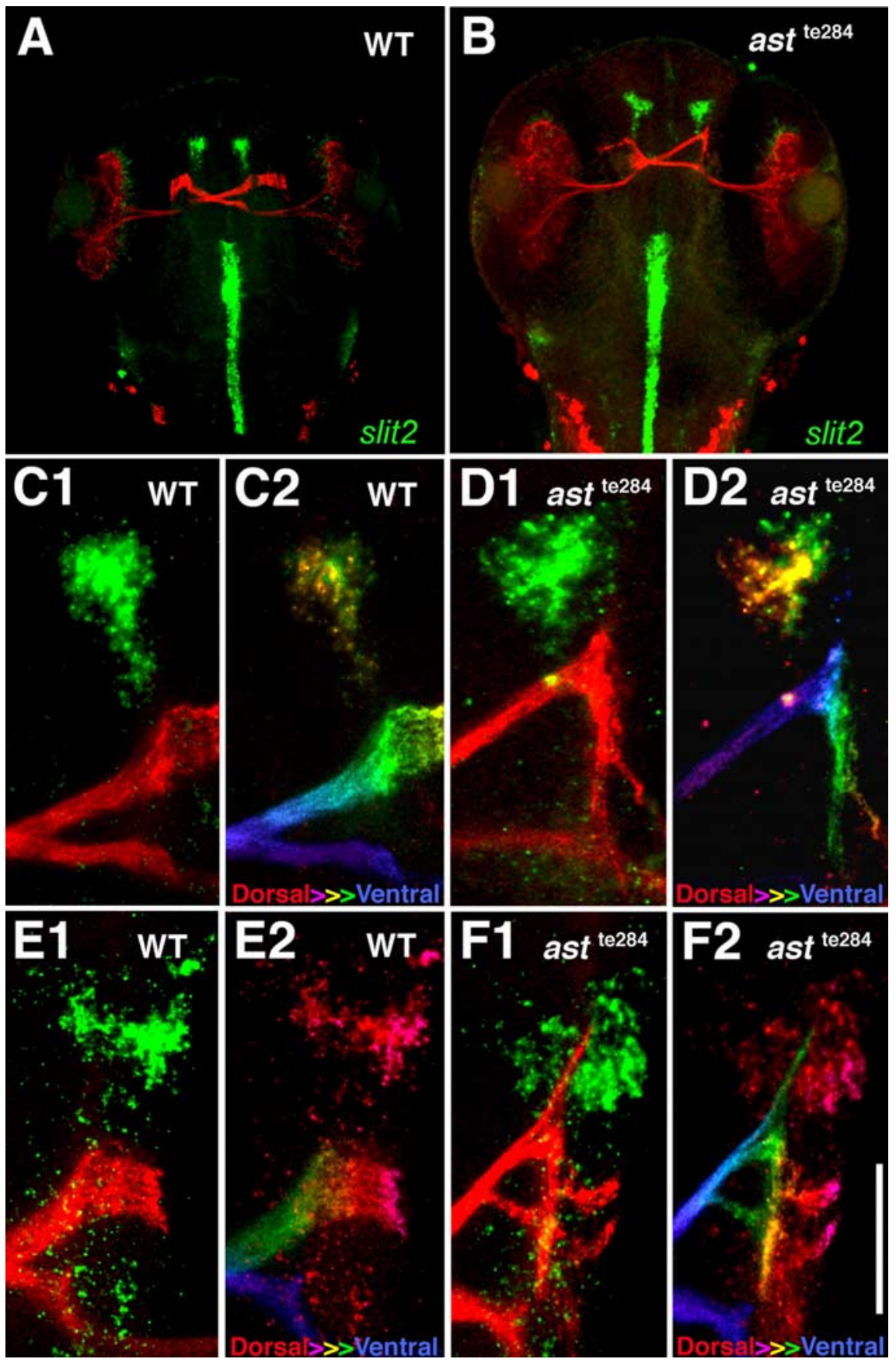

Figure 4. Retinal axons extend close to slit2 expressing cells in ast ${ }^{\text {te284 }}$ larvae. Retinal axons expressing GFP (red) and slit2 mRNA (green) in 54 hpf larvae that resulted from mating of parents heterozygous for both ast ${ }^{\text {te } 284}$ and Brn3c:GFP are shown. $A$, Phenotypically normal axons cross to the contralateral side and project dorsally. $\boldsymbol{B}$, Phenotypically astray axons frequently project anteriorly. $\mathbf{C 1}_{\text {, }}$ C2, A high power view of slitz expression (green) and retinal axons (red) in a phenotypically normal larva (C1) color coded so that both slit2 and retinal trajectories are blue at ventral levels and red at dorsal levels (C2). D1, slitz expression (green) and retinal axons (red) in a phenotypically astray larva. D2, The same image color-coded dorsal-to-ventral (red-to-violet). The abnormal anterior retinal projection is more ventral than normal and in close proximity to slit2 expressing cells. E1, E2, slit7a expression and retinal axons in a phenotypically normal larva (E1) color-coded by dorsal-to-ventral level (E2). $\mathbf{F 1}, \boldsymbol{F 2}$, slit1a expression (green) and retinal axons (red) in a phenotypically astray larva $(\boldsymbol{F} \mathbf{1})$ color coded by dorsal-to-ventral level (red to violet) $(\boldsymbol{F} 2)$. The abnormal anterior retinal projection extends just ventral to slit1a-expressing cells. Scale bar: (in F2) $A, B, 250 \mu \mathrm{m} ;(1-F 2,45 \mu \mathrm{m}$.

ing. Under normal conditions, slits expressed anteriorly to the chiasm would be expected to provide a strong source of repellent that prevents retinal axons from extending forward. When slit signaling is reduced, SDF1a expressed at the margin of the CNS anterior to the optic stalk may have the potential to attract retinal 

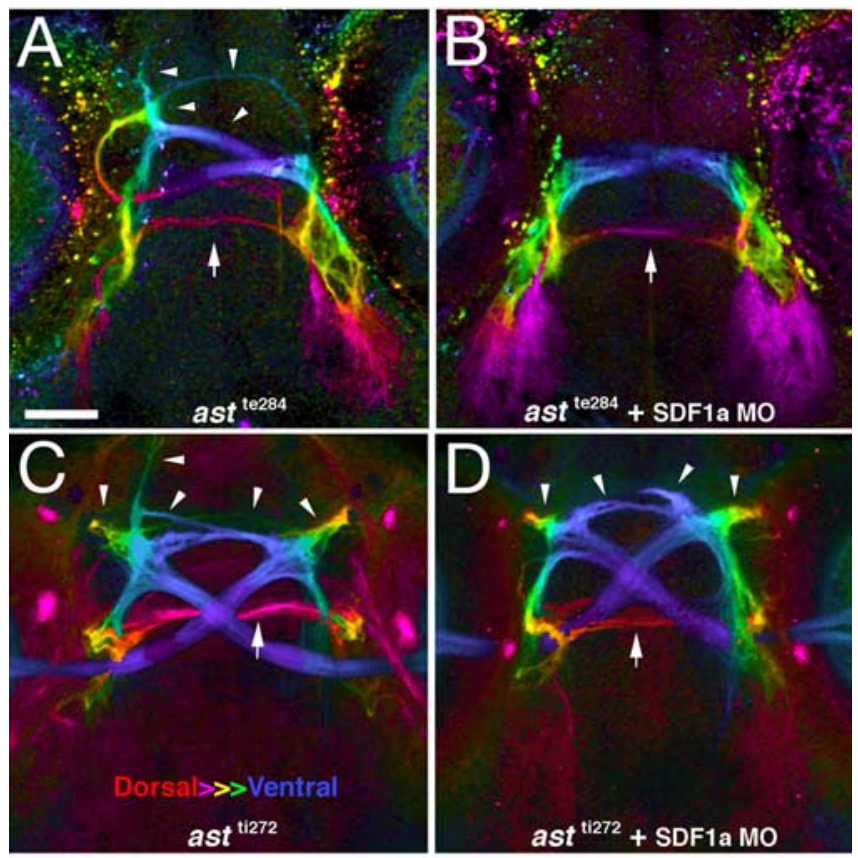

$E$

ast $^{\text {te284 }}$

$\mathrm{F}$

ast $^{\mathrm{ti272}}$
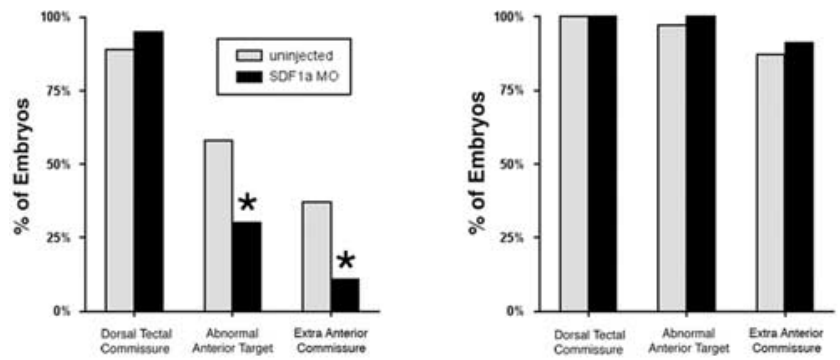

Figure 5. Reduced SDF1a signaling rescues abnormal anterior retinal projections in ast ${ }^{\text {te284 }}$ but not ast ${ }^{\text {ti272z }}$ larvae. $\boldsymbol{A}, \boldsymbol{B}$, Retinal axons expressing GFP are visualized in astray ${ }^{\text {te284 }}$ larvae $(\boldsymbol{A})$ and astray ${ }^{\text {te } 284}$ larvae $(\boldsymbol{B})$ injected with morpholino against SDF1a. Violet indicates the ventralmost depth whereas red indicates the dorsal-most depth. Both anterior-directed (arrow heads) and dorsal/posterior-located (arrows) misprojections are evident in astray larvae whereas posterior/dorsal errors were observed in SDF1a morphants. $C, D, R G C$ projections in astray ${ }^{\text {te272 }}$ larvae $(\boldsymbol{C})$ and SDF1a morpholino injected astray ${ }^{\text {te272 }}$ larvae $(\boldsymbol{D})$ were compared. The chiasm is shown in blue and arrowheads indicate anterior and arrows indicate posterior misprojections. Scale bar: (in $\boldsymbol{A}) \boldsymbol{A}-\boldsymbol{D}, 50 \mu \mathrm{m}$. $\boldsymbol{E}$, Percent of posterior dorsal tectal errors (first pair of bars) and anterior errors (second and third pairs of bars) are compared for astray larvae ( $n=65$; black bars) or astray larvae injected with SDF1a morpholino ( $n=44$; gray bars). SDF1a morpholino rescues anterior directed errors, but not dorsal errors. The differences between error rate between the uninjected and the injected embryos are significant for abnormal anterior targets and extra anterior commissures to the ${ }^{*} p<0.01$ level by Fisher's exact $t$ test. $\boldsymbol{F}, \mathrm{RGC}$ projections in rob02 null mutant (astray ${ }^{\text {te272 }}$ ) larvae ( $n=31$; black bars) and those injected with SDF1a morpholino ( $n=33$; gray bars) were also compared. None of the errors showed any significant difference between the two populations.

axons into ectopic trajectories. Knocking down SDF1a might then rescue astray embryos by preventing them from being diverted onto an alternative but normally inaccessible ectopic route.

Alternatively, SDF1a could be acting as an antirepellent rather than an attractant, significant only insofar as it reduces axonal responses to repellent cues. Under normal conditions, high slit expression anterior to the chiasm would prevent retinal axons from extending forward even though SDFla produced nearby lessens the effectiveness of slit. When slit signaling is reduced in intensity, however, the ability of SDF1a to make axons less re-
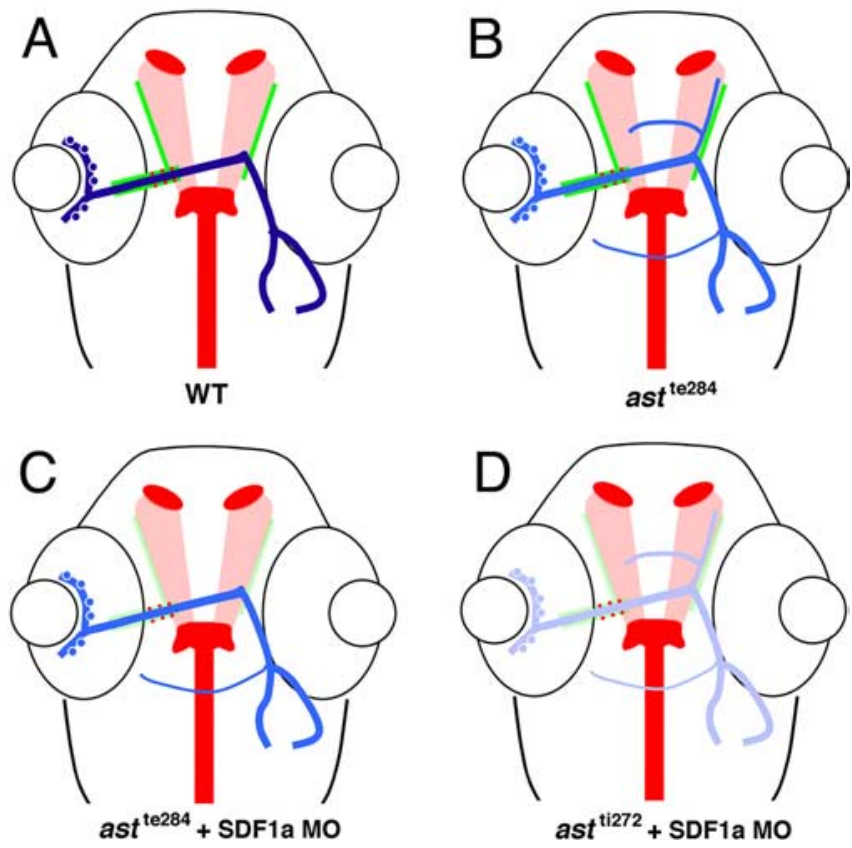

Figure 6. Summary of results. Schematic showing the trajectories of retinal axons in (shades of blue) and the expression patterns of slits (shades of red) and SDF1a (green). $\boldsymbol{A}$, In wild-type fish, retinal axons expressing normal levels of robo2 (navy blue) exit the eye through the optic stalk and enter the CNS along a path that expresses both SDF1a and slit2. After crossing the ventral midline at the optic chiasm, they extend to the lateral edge of the CNS where they grow dorsally and posteriorly toward the tecta. $\boldsymbol{B}$, Astray axons with reduced robo2 function (blue) project ectopically into more anterior regions after crossing the ventral midline, suggesting that their normal dorsal and posterior trajectory is in part determined by their avoidance of anterior concentrations of slits. C, Morpholinos against SDF1a (or CXCR4b) rescue anteriorly misprojecting retinal axons in embryos with reduced robo 2 function. $D$, Morpholinos against SDF1a do not rescue anteriorly misprojecting retinal axons in embryos with no robo2 function (pale blue).

sponsive to repellents would open the door to abnormal anteriorward extension.

Our finding that there is no rescue of a null ast $t^{\mathrm{ti272z}}$ allele of astray, in which slit/robo signaling is completely absent, supports the antirepellent rather than the attractant model for SDFla activity. If SDFla acts as an attractant that pulls retinal axons onto an incorrect pathway when slit signaling is reduced, then it should act similarly when all slit signaling is absent. Knocking down SDF1a signaling should then rescue both ast ${ }^{\mathrm{te} 284}$ and ast ${ }^{\mathrm{t} i 272 \mathrm{z}}$. However, we only observed rescue of the hypomorphic ast ${ }^{\text {te284 }}$ allele. Our interpretation of these findings is that the functional antagonism we see between SDF1 and slit signaling does not reflect integration of simultaneous SDF1-mediated attractive and slit-mediated repellent signals, but instead the ability of SDF1 to make axons less responsive to repellent activities without having an attractive activity of its own.

Both our in vivo and in vitro results are consistent with SDF1/ CXCR4-mediated signaling working antagonistically to slit/ robo-mediated repulsion during retinal axon pathfinding in the zebrafish embryo. Our results demonstrate that endogenous levels of SDF1 functionally antagonize the repellent effects of slit/ robo signaling in the developing retinal pathway. They suggest that, at least in some instances, SDFla signaling makes retinal axons less responsive to the repellent slit in vivo. Regardless of whether SDF1 acts as a traditional chemoattractant, or by reducing axonal responses to repellents, its expression in the optic stalk helps axons extend into an environment that might otherwise be precluded by the presence of powerful repellent cues. Our studies highlight the importance of having a proper balance between 
repulsive and modulatory signals during axon pathfinding in vivo.

\section{References}

Baier H, Klostermann S, Trowe T, Karlstrom RO, Nusslein-Volhard C, Bohoeffer F (1996) Genetic dissection of the retinotectal projection. Development 123:415-425.

Burrill JD, Easter SSJ (1994) Development of the retinofugal projections in the embryonic and larval zebrafish. J Comp Neurol 346:583-600.

Chalasani SH, Sabelko KA, Sunshine MJ, Littman DR, Raper JA (2003a) A chemokine, SDF-1, reduces the effectiveness of multiple axonal repellents and is required for normal axon pathfinding. J Neurosci 23:1360-1371.

Chalasani SH, Baribaud F, Coughlan CM, Sunshine MJ, Lee VM, Doms RW, Littman DR, Raper JA (2003b) The chemokine stromal cell-derived factor-1 promotes the survival of embryonic retinal ganglion cells. J Neurosci 23:4601-4612.

Chong SW, Emekyanov A, Gong Z, Korzh V (2001) Expression pattern of two zebrafish genes, cxcr4a and cxcr4b. Mech Dev 109:347-354.

Cohen A, Bray GM, Aguayo AJ (1994) Neurotrophin-4/5 (NT-4/5) increases adult rat retinal ganglion cell survival and neurite outgrowth in vitro. J Neurobiol 25:953-959.

Cohen J, Burne JF, McKinlay C, Winter J (1987) The role of laminin and the laminin/fibronectin receptor complex in the outgrowth of retinal ganglion cells. Dev Biol 122:407-418.

David NB, Sapede D, Saint-Etienne L, Thisse C, Thisse B, Dambly-Chaudiere C, Rosa FM, Ghysen A (2002) Molecular basis of cell migration in the fish lateral line: role of the chemokine receptor CXCR4 and of its ligand, SDF1. Proc Natl Acad Sci USA 99:16297-16302.

Deiner MS, Kennedy TE, Fazeli A, Serafini T, Tessier-Lavigne M, Sretavan DW (1997) Netrin-1 and DCC mediate axon guidance locally at the optic disc: loss of function leads to optic nerve hypoplasia. Neuron 19:575-589.

Doitsidou M, Reichman-Fried M, Stebler J, Koprunner M, Dorries J, Meyer D, Esguerra CV, Leung T, Raz E (2002) Guidance of primordial germ cell migration by the chemokine SDF-1. Cell 111:647-659.

Erskine L, Williams SE, Brose K, Kidd T, Rachel RA, Goodman CS, TessierLavigne M, Mason CA (2000) Retinal ganglion cell axons guidance in the mouse optic chiasm: expression and function of robos and slits. J Neurosci 20:4975-4982.

Fricke C, Lee JS, Geiger-Rudolph S, Bonhoeffer F, Chien CB (2001) astray, a zebrafish roundabout homolog required for retinal axon guidance. Science 292:507-510.

Goldberg JL, Vargas ME, Wang JT, Mandemakers W, Oster SF, Sretavan DW, Barres BA (2004) An oligodendrocyte lineage-specific semaphoring, Sema $5 \mathrm{~A}$, inhibits axon growth by retinal ganglion cells. J Neurosci 24:4989-4999.
Hutson LD, Chien CB (2002) Pathfinding and error correction by retinal axons: the role of astray/robo2. Neuron 33:205-217.

Karlstrom RO, Trowe T, Klostermann S, Baier H, Brand M, Crawford AD, Grunewald B, Haffter P, Hoffman H, Meyer SU, Muller BK, Richter S, van Eeden FJ, Nusslein-Volhard C, Bonhoeffer F (1996) Zebrafish mutations affecting retinotectal axon pathfinding. Development 123:427-438.

Knaut H, Werz C, Geisler R, Nusslein-Volhard C, Tubingen 2000 Screen Consortium (2003) A zebrafish homolog of the chemokine receptor, Cxcr4, is a primordial germ cell guidance receptor. Nature 421:279-282.

Li Q, Shirabe K, Kuwada JY (2004) Chemokine signaling regulated sensory cell migration in zebrafish. Dev Biol 269:123-136.

Li Q, Shirabe K, Thisse C, Thisse B, Okamoto H, Masai I, Kuwada JY (2005) Chemokine signaling guides axons within the retina in zebrafish. J Neurosci 25:1711-1717.

Monschau B, Kremoser C, Ohta K, Tanaka H, Kaneko T, Yamada T, Handwerker C, Hornberger MR, Loschinger J, Pasquale EB, Siever DA, Verderame MF, Muller BK, Bonhoeffer F, Drescher U (1997) Shared and distinct functions of RAGS and ELF-1 in guiding retinal axons. EMBO J 16:1258-1267.

Mullins MC, Hammerschmidt M, Haffter P, Nusselein-Volhard C (1994) Large-scale mutagenesis in the zebrafish: in search of genes controlling development in the vertebrate. Curr Biol 4:189-202.

Nagasawa T, Kikutani H, Kishimoto T (1994) Molecular cloning and structure of a pre-B-cell growth stimulating factor. Proc Natl Acad Sci USA 91:2305-2309.

Nasevicius A, Ekker SC (2000) Effective targeted gene "knockdown" in zebrafish. Nat Genet 26:216-220.

Niclou SP, Jia L, Raper JA (2000) slit2 is a repellent for retinal ganglion cell axons. J Neurosci 20:4962-4974

Plump AS, Erskine L, Sabatier C, Brose K, Epstein CJ, Goodman CS, Mason CA, Tessier-Lavogne M (2002) slit1 and slit2 cooperate to prevent premature midline crossing of retinal axons in the mouse visual system. Neuron 33:219-232.

Sakai JA, Halloran MC (2006) Semaphorin 3d guides laterality of retinal ganglion cell projections in zebrafish. Development 133:1035-1044.

Stuermer CA (1988) Retinotopic organization of the developing retinotectal projection in the zebrafish embryo. J Neurosci 8:4513-4530.

Tessier-Lavigne M, Goodman CS (1996) The molecular biology of axon guidance. Science 274:1123-1133.

Williams SE, Mann F, Erskine L, Sakurai T, Wei S, Rossi DJ, Gale NW, Holt CE, Mason CA, Henkemeyer M (2003) Ephrin-B2 and EphB1 mediate retinal axon divergence at the optic chaism. Neuron 39:919-935.

Xiao T, Roeser T, Staub W, Baier H (2005) A GFP based genetic screen reveals mutations that disrupt the architecture of the zebrafish retinotectal projection. Development 132:2955-2967.

Yu WT, Bargmann CI (2001) Dynamic regulation of axon guidance. Nat Neurosci 4:1169-1176. 